\title{
Biosynthetic Pathways and the Role of the Mas Receptor in the Effects of Angiotensin-(1-7) in Smooth Muscles
}

\author{
Marcio Augusto Fressatto de Godoy, ${ }^{1}$ Larissa Pernomian, ${ }^{2}$ \\ Ana Maria de Oliveira, ${ }^{3}$ and Satish Rattan ${ }^{1}$ \\ ${ }^{1}$ Division of Gastroenterology \& Hepatology, Department of Medicine, Jefferson Medical College, \\ Thomas Jefferson University, Philadelphia, PA, USA \\ ${ }^{2}$ Laboratory of Pharmacology, Department of Pharmacology, Faculty of Medicine from Ribeirão Preto, \\ University of São Paulo (USP), Avenida do Café s/n, 14040-903 Ribeirão Preto, SP, Brazil \\ ${ }^{3}$ Laboratory of Pharmacology, Department of Physics and Chemistry, Faculty of Pharmaceutical Sciences from Ribeirão Preto, \\ University of São Paulo (USP), Avenida do Café s/n, 14040-903 Ribeirão Preto, SP, Brazil
}

Correspondence should be addressed to Satish Rattan, Satish.Rattan@Jefferson.edu

Received 27 July 2011; Revised 19 September 2011; Accepted 22 September 2011

Academic Editor: Anderson J. Ferreira

Copyright (C) 2012 Marcio Augusto Fressatto de Godoy et al. This is an open access article distributed under the Creative Commons Attribution License, which permits unrestricted use, distribution, and reproduction in any medium, provided the original work is properly cited.

\begin{abstract}
Ang-(1-7) is produced via degradation of Ang II by the human angiotensin converting enzyme, also known as ACE2. In the cardiovascular system, Ang-(1-7) has been shown to produce effects that are opposite to those of Ang II. These include smooth muscle relaxation and cardioprotection. While the roles of Ang-(1-7) in other systems are currently topic of intense research, functional data suggest a relaxation action in gastrointestinal smooth muscles in a way that corroborates the results obtained from vascular tissues. However, more studies are necessary to determine a relevant role for Ang-(1-7) in the gastrointestinal system. The Ang-(1-7) actions are mediated by a distinct, functional, Ang-(1-7) receptor: the Mas receptor as shown by diverse studies involving site-specific binding techniques, selective antagonists, and targeted gene deletion. This paper provides an overview of the functional role and the molecular pathways involved in the biosynthesis and activity of Ang-(1-7) in diverse systems.
\end{abstract}

\section{A Brief Historical Overview}

Until the late 80 s, it was thought that most of the biological activity of angiotensin peptides was based on their interaction with the $\mathrm{AT}_{1}$ receptor at the $\mathrm{C}$-terminal side chain of a phenylalanine residue in the position $8\left(\mathrm{Phe}^{8}\right)[1,2]$. Consequently, it was assumed that fragments of Ang II lacking the C-terminal $\mathrm{Phe}^{8}$ were biologically inactive [3]. A number of studies have shown that the $\mathrm{N}$-terminal heptapeptide angiotensin-(1-7) [Ang-(1-7)], also named as des$\left[\mathrm{Phe}^{8}\right]$-angiotensin II [4], lacked vasopressor effect [5, 6], aldosterone release activity [5], and central dipsogenic action [7].

However, in 1988, Santos and coworkers showed that Ang-(1-7) was produced as the main metabolite of angiotensin I (Ang I) in dog brainstem and spinal cord, which is produced even in the presence of angiotensin-converting enzyme (ACE) inhibitors, suggesting an ACE-independent route [8]. Further studies have shown that Ang-(1-7) stimulates arginine vasopressine (AVP) release from the rat hypothalamo-neurohypophysial system (HNS) with potency comparable to angiotensin II (Ang II) [9]. These findings triggered general scientific interest in the area with a series of studies involving site-specific, functional antagonism, and targeted-gene deletion that, among other techniques, have resulted in the identification of ACE2 and of the Mas receptor as the main agents responsible for the biosynthesis and actions of Ang-(1-7) at the molecular level.

This paper provides an overview of the molecular pathways involved in the biosynthesis and activity of Ang(1-7) in the vascular and gastrointestinal systems with emphasis on their smooth muscle structures, and the limited availability of such information in the gastrointestinal tract. 


\section{Biosynthesis and Degradation of Ang-(1-7)}

ACE2 is the enzyme responsible for the biosynthesis of Ang(1-7). ACE2 is a membrane-associated zinc metalloprotease and a homologue of the human ACE isoforms, which is highly expressed in several tissues such as human heart, kidney, lungs, and testis $[10,11]$. There is also evidence for the presence of ACE2 in smooth muscles: in the cardiovascular system, studies have shown the expression of ACE2 in the media of thoracic aorta and common carotid arteries from spontaneously hypertensive rats (SHRs) [12]. In line with these studies, ACE2 has also been shown to be expressed in vascular smooth muscle cells (VSMCs) isolated from rat aorta $[13,14]$, and in renal and mesenteric arteries from spontaneously hypertensive stroke-prone rats (SHRSP) [15]. In the gastrointestinal system, studies suggest a role for local biosynthesis of Ang-(1-7). These include an inhibitory effect in the basal tone of the internal anal sphincter [16] and the identification of ACE2 mRNA in the stomach wall of rats [17]. In addition, studies using quantitative realtime polymerase chain reaction (QRT-PCR) have made the novel observation that ACE 2 shows levels of expression in the gastrointestinal system that are comparable to those in the cardiovascular system. Particularly high levels of ACE2 have been found in duodenum, jejunum, ileum, caecum, and colon. Therefore, consideration should also be given to a potential role for ACE2 in gastrointestinal physiology and pathophysiology [18]. However, other than this, there is relatively scarce information about formation and actions of Ang-(1-7) in the gastrointestinal system.

Despite its homology with ACE, ACE2 is functionally different as it acts as a C-terminal carboxypeptidase rather than a C-terminal dipeptidase by cleaving the C-terminal of a residue of Leu in the structure of Ang I or a residue of Phe in Ang II. This activity, respectively, generates angiotensin(1-9) [Ang-(1-9)] [10, 11] or Ang-(1-7) [11, 19].

There are also pharmacological differences between ACE and ACE2. ACE2 is insensitive to classic ACE inhibitors such as lisinopril [10,11], enalaprilat, or captopril [11] as well as other ACE inhibitors [20]. The differential sensitivity to ACE inhibitors results from amino acid substitutions in the substrate binding site from ACE2. Studies show that ACE2 has an Arginine (Arg) residue in the position 273 instead of the Glutamine (Gln) residue normally found in ACE [21]. This substitution allosterically impairs the interaction between ACE2 and classic ACE inhibitors because of the larger size of $\mathrm{Arg}^{273}$, which limits the size of the $\mathrm{S}_{2}{ }^{\prime}$ substrate subsite in ACE2 [21].

As mentioned above, ACE2 catalyzes the conversion of both Ang I and Ang II into smaller fragments. Donoghue et al. [10] first showed that ACE2 is able to hydrolyze Ang I. Following this, Vickers et al. [19] showed that ACE2 more efficiently hydrolyzes Ang II than Ang I since the latter is only partially hydrolyzed. ACE2 and the resulting formation of Ang-(1-7) play an important role in the cardiovascular system. Studies by Igase et al. [12] have shown that blockade of $\mathrm{AT}_{1}$ receptors in the media layer of thoracic aortas isolated from SHR resulted in significant increases in the expression of ACE2 at the mRNA and protein levels. This effect was consistently associated with increased levels of Ang-(1-7), suggesting that ACE2 generates Ang-(1-7) locally. Corroborating these results, studies by Lavrentyev and Malik [14] have found that reduction in ACE2 expression is followed by a reduction in the cellular levels of Ang-(1-7) in rat aorta VSMC. Together, these studies suggest the involvement of ACE2 in the generation of Ang-(1-7) within the VSMC. Similar results have been found in human coronary vessels [22] and rat stomach wall [17].

In addition to ACE2, there are other enzymes that have been reported to release Ang-(1-7). Endopeptidases are the first example and have been implicated in the biosynthesis of Ang-(1-7) mainly from Ang I. As an example, prolyl endopeptidase converts Ang I into Ang-(1-7) as shown in NG108-15 neuroblastoma versus glioma hybrid cells [23] and in endothelial cells from human and bovine aorta as well as in umbilical veins [24]. As another example, neutral endopeptidase (NEP or neprilysin) accounts for the generation of most of the circulating Ang-(1-7) derived from Ang I as suggested by studies in SHR and Wistar-Kyoto (WK) rats [25]. In addition, thimet oligopeptidase (EC 3.4.24.15) forms Ang-(1-7) from Ang I in VSMC [26]. The catalytic action of these peptidases is responsible for the efficiency of the Ang I-dependent pathways in the biosynthesis of Ang-(17) because ACE2 hydrolyzes Ang II rather than Ang I [19, 20].

ACE has also been reported to be able to release Ang-(1$7)$. Even though it does not catalyze the generation of Ang(1-7) from Ang I or Ang II, ACE is able to cleave Ang-(1-9) leading to the generation of Ang-(1-7) [20]. Furthermore, ACE is an important component in the catabolism of Ang$(1-7)$.

The first evidence that pointed a potential role for ACE in the degradation of Ang-(1-7) came from the findings obtained by Kohara et al. [27]. In this study, the authors observed that the chronic treatment of SHR or WK rats with the ACE inhibitors ceranopril or lisinopril augmented the circulating levels of Ang-(1-7). Corroborating with Kohara et al. [27], Luque et al. [28] showed that the chronic therapy of essential hypertensive subjects with captopril reduced the diastolic blood pressure without changing the plasma levels of Ang II while increasing the levels of Ang-(1-7). Taken together, these findings suggest a potential role for ACE in the degradation of Ang-(1-7), which may be involved in the antihypertensive effects from ACE inhibitors.

Indeed, in vitro experiments obtained by Chappell et al. [29] showed that ACE cleaves the Isoleucine (Ile $)^{5}-\mathrm{His}^{6}$ bond of Ang-(1-7) with a high specificity constant. The hydrolysis of Ang-(1-7) catalyzed by ACE involves the N-domain of the enzyme [30] and leads to the formation of angiotensin-(1-5) [Ang-(1-5)] [29].

\section{Effects Elicited by Ang-(1-7)}

Studies show that Ang-(1-7) has a cardioprotective role mainly attributable to counteraction of the Ang II effects, which contribute to maintaining the vascular homeostasis and attenuating the progression of atherogenesis [31, 32] among other effects as further explained below. 
Campagnole-Santos et al. [33] were the first to demonstrate the vascular effects induced by Ang-(1-7). The authors observed that the injection of Ang-(1-7) in the rat dorsal motor nucleus of the vagus elicited a centrally mediated hypotension similar to that induced by Ang II. Later, the authors showed that intracerebroventricular injections of Ang-(1-7) increased the baroreflex sensitivity for the control of the heart rate in conscious rats, suggesting that Ang(1-7) facilitates the baroreflex by inducing depressor effects into the dorsal medulla [34]. In this context, Benter et al. [35] found that systemic injections of Ang-(1-7) transiently reduced the systolic pressure in SHR, suggesting a potential counterregulatory effect of Ang-(1-7) on the pressor effects elicited by the main agents that increase the total peripheral resistance during hypertension, like Ang II and $\alpha_{1}$-adrenergic agonists. Later, the authors confirmed their hypothesis [36] by showing that the intravenous administration of Ang-(17) reduced the pressor responsiveness to the $\alpha_{1}$-adrenergic agonist phenylephrine, and to Ang II, and improved the sensitivity of the reflex control of the heart rate in SHR. These results were suggestive that Ang-(1-7) activated antihypertensive mechanisms.

Additional studies showed that Ang-(1-7) was responsible for the hypotensive effects of ACE inhibitors like lisinopril and losartan. Experiments using monoclonal antibodies selective for Ang-(1-7) showed that the antihypertensive effects of lisinopril and losartan were reversed by scavenging the circulating Ang-(1-7) in SHR [29, 30, 37, 38]. Following diverse studies showed that Ang-(1-7) has a relaxation effect of its own. Meng et al. showed that Ang-(1-7) caused a mild dilatation of cerebral arterioles via a mechanism that involves the release of cyclooxygenases (COX) metabolites [39]. Pörsti et al. [40] demonstrated that Ang-(1-7) induced an endothelium- and nitric oxide synthase (NOS) metabolitesmediated relaxation in porcine coronary arteries. Similar findings were obtained by Brosnihan et al. [41], who showed an endothelium- and NOS metabolites-mediated relaxation evoked by Ang-(1-7) in canine coronary artery. Comparable results were found in other animal species using a number of tissues suggesting that Ang-(1-7) has a widespread relaxation effect in the cardiovascular system via $\mathrm{NO}$ release [42-44]. In the vascular system, the counterregulatory actions of Ang-(1-7) over the effects produced by Ang II occur at the molecular level. Sampaio et al. [45] have shown that Ang-(1-7) inhibits the assembly and activation of $\mathrm{NAD}(\mathrm{P}) \mathrm{H}$ oxidase induced by Ang II by inhibiting Ang II-induced phosphorylation of p47phox, which is crucial to the $\mathrm{NAD}(\mathrm{P}) \mathrm{H}$ oxidase activation. This Ang II-mediated mechanism results in $\mathrm{O}_{2}{ }^{-}$generation [46], $\mathrm{NO}$ inactivation [47], and has been correlated with diabetes. During diabetes mellitus, the vascular endothelium is an important source of $\mathrm{NAD}(\mathrm{P}) \mathrm{H}$ oxidase-derived $\mathrm{O}_{2}{ }^{-}$, which is involved in the reduction of endothelial $\mathrm{NO}$ availability and in the consequent endothelial dysfunction [47, 48]. Therefore, the counteractive effects by Ang-(1-7) over Ang II make it a potentially important therapeutic target to attenuate the endothelial dysfunction and in treating diabetes mellitus.
Ang-(1-7) also has antiproliferative effects in the cardiovascular system. Freeman et al. [49] have shown that Ang-(17) inhibits incorporation of mitogen-stimulated thymidine in rat aortic VSMC thus inhibiting cellular growth. The mechanism was characterized by Tallant and Clark [50], who showed that Ang-(1-7) attenuates the mitogenic activity of MAPK by a cAMP-dependent protein kinase (PKA), which is activated after a $\mathrm{PGI}_{2}$-mediated increase in cAMP production. In addition, Strawn et al. [51] have shown that Ang-(1-7) treatment reduced the DNA synthesis and the cross-sectional area of neointima in rat carotid artery injured by balloon catheter. In line with these results, Langeveld et al. [52] observed that Ang-(1-7) treatment reduces the neointimal thickness in rat abdominal aorta after stent implantation and restores the impaired endothelial function. Many other studies also support the concept that Ang-(1-7) plays an important role in neointimal re-endothelization and inhibition of neointimal formation and restenosis [52-54].

Studies also provide evidence for a role for Ang-(1-7) in reducing thrombus formations. Experiments in rat vena cava have shown that Ang-(1-7) reduces the thrombus weight and platelet adhesion to fibrilar collagen by a mechanism involving NOS and COX metabolites [55]. In addition, Tesanovic et al. [56] found that the chronic treatment with Ang-(1-7) reduced the development of atherosclerotic lesion in ApoE (-/-) and high-fat diet-fed mice, followed by an increase in the local expression of $e$ NOS. These findings have opened new perspectives on the promising vasculo- and atheroprotective effects of Ang-(1-7) suggesting therapeutic potentials for the atherosclerosis, thrombosis, and atherothrombosis.

\section{Mas Receptor}

The Mas receptor was first cloned and sequenced by Young et al. [57] in cotransfected NIH 3T3 cells from nude mice and revealed a very hydrophobic protein, containing seven potential transmembrane domains.

A connection between the Mas receptor and Ang-(17) was first established by Santos et al. [58]. Using binding studies performed in kidney sections from wild-type and Mas-deficient mice, the authors demonstrated that Ang-(17) binding was absent in kidneys from Mas-deficient mice, but preserved in wild-type membranes. In addition, the authors also showed that Ang-(1-7) binding was preserved in membranes isolated from $\mathrm{AT}_{1}$ - or $\mathrm{AT}_{2}$-deficient animals, suggesting a twofold conclusion: (1) that Ang-(1-7) has limited interactions with $\mathrm{AT}_{1}$ and $\mathrm{AT}_{2}$ receptors and (2) that it mainly binds to Mas receptors [58]. These results were confirmed by further studies showing high affinity sites for Ang-(1-7) in Mas receptors. The results also showed that the Mas receptor has very low affinity for $\mathrm{AT}_{1}$ or $\mathrm{AT}_{2}$ ligands, excluding the hypothesis that Ang-(1-7) would directly interact with $\mathrm{AT}_{1}$ or $\mathrm{AT}_{2}$ receptors [58].

The first evidence for a functional role for the Mas receptor as the mediator of the Ang-(1-7) effects in the vascular system was also provided by Santos et al. [58]. The authors showed that the relaxation induced by Ang(1-7) in mouse aorta was absent in Mas-deficient mice. 
The authors were also the first group to develop a selective antagonist for the Mas receptor called D-Ala ${ }^{7}$-Ang-(1-7) also known as A-779 [58, 59]. These findings fostered publication of many functional studies supporting a role for Mas receptors in the effects induced by Ang-(1-7). These include indirect (centrally mediated) antihypertensive effects [60] and local effects at the cellular [61] and molecular [44] levels. Tirapelli et al. [61] have shown that A-779 inhibited vasorelaxant effects caused by Ang-(1-7) in rat carotid artery in a concentration-dependent fashion. Sampaio et al. [44] demonstrated that A-779 blocks the phosphorylation of eNOS induced by Ang-(1-7) in human aortic endothelial cells, suggesting the involvement of Mas receptors in this effect. A-779 also blocked the inhibitory effect of Ang-(17) on VSMC growth, suggesting the participation of Mas receptors in the antiproliferative action of Ang-(1-7) [62].

There is also functional evidence directly supporting a role for Mas receptors in the antithrombotic effect of Ang-(1-7). A-779 dose-dependently inhibits the Ang-(17)-induced reduction in venous thrombus weight [55]. In addition, A-779 reduces the development of atherosclerotic lesion by Ang-(1-7) in mice. Interestingly, this effect was more evident when A-779 was combined with the $\mathrm{AT}_{2}$ antagonists, suggesting an important interaction between Mas and $\mathrm{AT}_{2}$ receptors in mediating the atheroprotective effect of Ang-(1-7) [56].

Multipronged studies also show a potential role for Mas receptors and Ang-(1-7) in the regulation of gastrointestinal smooth muscle motility. Studies by De Godoy et al. in Dr. Rattan's laboratory [16] have shown that Ang-(1-7) dosedependently reduces the basal tone of spontaneously contracted internal anal sphincter of rats. The studies also show that this relaxation effect is abolished by A-779. In addition, the studies have shown that A-779 had no significant effect on the contractile response of Ang II [16]. Whether Ang(1-7) has an important role in the gastrointestinal system remains to be determined.

In closing, Ang-(1-7) relaxes smooth muscles via interactions with the Mas receptor that elicit well-known molecular mechanisms of relaxation such as the release of NO. Limited studies suggest a potential role for Ang-(1-7) in regulating motility of gastrointestinal smooth muscles, but additional studies are necessary to further determine Ang-(1-7) actions and therapeutic potentials in the gastrointestinal system. On the other hand, the effects of Ang-(1-7) in the cardiovascular system are better understood and provide strong evidence for the cardiovascular protective action that can be selectively modulated via Mas ligands and have important therapeutic implications for human therapy.

\section{Acknowledgments}

The work was supported by National Institutes of Diabetes and Digestive and Kidney Diseases Grant DK-35385 and an institutional grant from Thomas Jefferson University.

\section{References}

[1] T. L. Goodfriend, "Angiotensins. A family that grows from within," Hypertension, vol. 17, no. 2, pp. 139-140, 1991.
[2] P. B. Timmermans, P. C. Wong, A. T. Chiu et al., "Angiotensin II receptors and angiotensin II receptor antagonists," Pharmacological Reviews, vol. 45, no. 2, pp. 205-251, 1993.

[3] M. T. Schiavone, M. C. Khosla, and C. M. Ferrario, "Angiotensin-[1-7]: evidence for novel actions in the brain," Journal of Cardiovascular Pharmacology, vol. 16, no. 4, supplement, pp. S19-S24, 1990.

[4] K. Kohara, K. B. Brosnihan, M. C. Chappell, M. C. Khosla, and C. M. Ferrario, "Angiotensin-(1-7). A member of circulating angiotensin peptides," Hypertension, vol. 17, no. 2, pp. 131$138,1991$.

[5] I. H. Page and F. M. Bumpus, "Angiotensin," Physiological reviews, vol. 41, pp. 331-390, 1961.

[6] J. A. Tonnaer, G. M. H. Engels, and V. M. Wiegant, "Proteolytic conversion of angiotensins in rat brain tissue," European Journal of Biochemistry, vol. 131, no. 2, pp. 415-421, 1983.

[7] J. T. Fitzsimons, "The effect on drinking of peptide precursors and of shorter chain peptide fragments of angiotensin II injected into the rat's diencephalon," Journal of Physiology, vol. 214, no. 2, pp. 295-303, 1971.

[8] R. A. S. Santos, K. B. Brosnihan, M. C. Chappell et al., "Converting enzyme activity and angiotensin metabolism in the dog brainstem," Hypertension, vol. 11, no. 2, pp. I153-I157, 1988.

[9] M. T. Schiavone, R. A. S. Santos, K. B. Brosnihan, M. C. Khosla, and C. M. Ferrario, "Release of vasopressin from the rat hypothalamo-neurohypophysial system by angiotensin(1-7) heptapeptide," Proceedings of the National Academy of Sciences of the United States of America, vol. 85, no. 11, pp. 4095-4098, 1988.

[10] M. Donoghue, F. Hsieh, E. Baronas et al., "A novel angiotensin-converting enzyme-related carboxypeptidase (ACE2) converts angiotensin I to angiotensin 1-9," Circulation research, vol. 87, no. 5, pp. E1-E9, 2000.

[11] S. R. Tipnis, N. M. Hooper, R. Hyde, E. Karran, G. Christie, and A. J. Turner, "A human homolog of angiotensinconverting enzyme: cloning and functional expression as a captopril-insensitive carboxypeptidase," Journal of Biological Chemistry, vol. 275, no. 43, pp. 33238-33243, 2000.

[12] M. Igase, W. B. Strawn, P. E. Gallagher, R. L. Geary, and C. M. Ferrario, "Angiotensin II at1 receptors regulate ACE2 and angiotensin-(1-7) expression in the aorta of spontaneously hypertensive rats," American Journal of Physiology, vol. 289, no. 3, pp. H1013-H1019, 2005.

[13] E. N. Lavrentyev, A. M. Estes, and K. U. Malik, "Mechanism of high glucose-induced angiotensin II production in rat vascular smooth muscle cells," Circulation Research, vol. 101, no. 5, pp. 455-464, 2007.

[14] E. N. Lavrentyev and K. U. Malik, "High glucose-induced Nox1-derived superoxides downregulate PKC- $\beta$ II, which subsequently decreases ACE2 expression and ANG(1-7) formation in rat VSMCs," American Journal of Physiology, vol. 296, no. 1, pp. H106-H118, 2009.

[15] B. Rentzsch, M. Todiras, R. Iliescu et al., "Transgenic angiotensin-converting enzyme 2 overexpression in vessels of SHRSP rats reduces blood pressure and improves endothelial function," Hypertension, vol. 52, no. 5, pp. 967-973, 2008.

[16] M. A. F. De Godoy, S. Dunn, and S. Rattan, "Evidence for the role of angiotensin II biosynthesis in the rat internal anal sphincter tone," Gastroenterology, vol. 127, no. 1, pp. 127-138, 2004. 
[17] R. Olszanecki, J. Madej, M. Suski, A. Gebska, B. BujakGizycka, and R. Korbut, "Angiotensin metabolism in rat stomach wall: prevalence of angiotensin-1-7 formation," Journal of Physiology and Pharmacology, vol. 60, no. 1, pp. 191-196, 2009.

[18] D. Harmer, M. Gilbert, R. Borman, and K. L. Clark, "Quantitative mRNA expression profiling of ACE 2, a novel homologue of angiotensin converting enzyme," FEBS Letters, vol. 532, no. 1-2, pp. 107-110, 2002.

[19] C. Vickers, P. Hales, V. Kaushik et al., "Hydrolysis of biological peptides by human angiotensin-converting enzyme-related carboxypeptidase," Journal of Biological Chemistry, vol. 277, no. 17, pp. 14838-14843, 2002.

[20] G. I. Rice, D. A. Thomas, P. J. Grant, A. J. Turner, and N. M. Hooper, "Evaluation of angiotensin-converting enzyme (ACE), its homologue ACE2 and neprilysin in angiotensin peptide metabolism," Biochemical Journal, vol. 383, no. 1, pp. 45-51, 2004.

[21] P. Towler, B. Staker, S. G. Prasad et al., "ACE2 X-ray structures reveal a large hinge-bending motion important for inhibitor binding and catalysis," Journal of Biological Chemistry, vol. 279, no. 17, pp. 17996-18007, 2004.

[22] L. S. Zisman, G. E. Meixell, M. R. Bristow, and C. C. Canver, "Angiotensin-(1-7) formation in the intact human heart: in vivo dependence on angiotensin II as substrate," Circulation, vol. 108, no. 14, pp. 1679-1681, 2003.

[23] M. C. Chappell, E. A. Tallant, K. B. Brosnihan, and C. M. Ferrario, "Processing of angiotensin peptides by NG108-15 neuroblastoma x glioma hybrid cell line," Peptides, vol. 11, no. 2, pp. 375-380, 1990.

[24] R. A. S. Santos, K. B. Brosnihan, D. W. Jacobsen, P. E. DiCorleto, and C. M. Ferrario, "Production of angiotensin(1-7) by human vascular endothelium," Hypertension, vol. 19, no. 2, supplement, pp. II56-II61, 1992.

[25] K. Yamamoto, M. C. Chappell, K. B. Brosnihan, and C. M. Ferrario, "In vivo metabolism of angiotensin I by neutral endopeptidase (EC 3.4.24.11) in spontaneously hypertensive rats," Hypertension, vol. 19, no. 6, pp. 692-696, 1992.

[26] M. C. Chappell, E. A. Tallant, K. B. Brosnihan, and C. M. Ferrario, "Conversion of angiotensin i to angiotensin-(1-7) by thimet oligopeptidase (EC 3.4.24.15) in vascular smooth muscle cells," Journal of Vascular Medicine and Biology, vol. 5, no. 4, pp. 129-137, 1994.

[27] K. Kohara, K. B. Brosnihan, and C. M. Ferrario, "Angiotensin (1-7) in the spontaneously hypertensive rat," Peptides, vol. 14, no. 5, pp. 883-891, 1993.

[28] M. Luque, P. Martin, N. Martell, C. Fernandez, K. B. Brosnihan, and C. M. Ferrario, "Effects of captopril related to increased levels of prostacyclin and angiotensin-(1-7) in essential hypertension," Journal of Hypertension, vol. 14, no. 6, pp. 799-805, 1996.

[29] M. C. Chappell, N. T. Pirro, A. Sykes, and C. M. Ferrario, "Metabolism of angiotensin-(1-7) by angiotensin-converting enzyme," Hypertension, vol. 31, no. 1, pp. 362-367, 1998.

[30] P. A. Deddish, B. Marcic, H. L. Jackman, H. Z. Wang, R. A. Skidgel, and E. G. Erdös, "N-domain-specific substrate and c-domain inhibitors of angiotensin- converting enzyme angiotensin-(1-7) and keto-ace," Hypertension, vol. 31, no. 4, pp. 912-917, 1998.

[31] F. Lovren, Y. Pan, A. Quan et al., "Angiotensin converting enzyme-2 confers endothelial protection and attenuates atherosclerosis," American Journal of Physiology, vol. 295, no. 4, pp. H1377-H1384, 2008.
[32] M. C. Thomas, R. J. Pickering, D. Tsorotes et al., "Genetic Ace2 deficiency accentuates vascular inflammation and atherosclerosis in the ApoE knockout mouse," Circulation Research, vol. 107, no. 7, pp. 888-897, 2010.

[33] M. J. Campagnole-Santos, D. I. Diz, R. A. S. Santos, M. C. Khosla, K. B. Brosnihan, and C. M. Ferrario, "Cardiovascular effects of angiotensin-(1-7) injected into the dorsal medulla of rats," American Journal of Physiology, vol. 257, no. 1, pp. H324H329, 1989.

[34] M. J. Campagnole-Santos, S. B. Heringer, E. N. Batista, M. C. Khosla, and R. A. S. Santos, "Differential baroreceptor reflex modulation by centrally infused angiotensin peptides," American Journal of Physiology, vol. 263, no. 1, pp. R89-R94, 1992.

[35] I. F. Benter, C. M. Ferrario, M. Morris, and D. I. Diz, "Antihypertensive actions of angiotensin-(1-7) in spontaneously hypertensive rats," American Journal of Physiology, vol. 269, no. 1, pp. H313-H319, 1995.

[36] I. F. Benter, D. I. Diz, and C. M. Ferrario, "Pressor and reflex sensitivity is altered in spontaneously hypertensive rats treated with angiotensin-(1-7)," Hypertension, vol. 26, no. 6, pp. 1138-1144, 1995.

[37] S. N. Iyer, M. C. Chappell, D. B. Averill, D. I. Diz, and C. M. Ferrario, "Vasodepressor actions of angiotensin-(1-7) unmasked during combined treatment with lisinopril and losartan," Hypertension, vol. 31, no. 2, pp. 699-705, 1998.

[38] P. Li, M. C. Chappell, C. M. Ferrario, and K. B. Brosnihan, "Angiotensin-(1-7) augments bradykinin-induced vasodilation by competing with ACE and releasing nitric oxide," Hypertension, vol. 29, no. 1, pp. 394-400, 1997.

[39] W. Meng, D. W. Busija, and W. G. Mayhan, "Comparative effects of angiotensin-(1-7) and angiotensin II on piglet pial arterioles," Stroke, vol. 24, no. 12, pp. 2041-2045, 1993.

[40] I. Pörsti, A. T. Barra, R. Busse, and M. Hecker, "Release of nitric oxide by angiotensin-(1-7) from porcine coronary endothelium: implications for a novel angiotensin receptor," British Journal of Pharmacology, vol. 111, no. 3, pp. 652-654, 1994.

[41] K. B. Brosnihan, P. Li, and C. M. Ferrario, "Angiotensin-(17) dilates canine coronary arteries through kinins and nitric oxide," Hypertension, vol. 27, no. 3, pp. 523-528, 1996.

[42] Y. Ren, J. L. Garvin, and O. A. Carretero, "Vasodilator action of angiotensin-(1-7) on isolated rabbit afferent arterioles," Hypertension, vol. 39, no. 3, pp. 799-802, 2002.

[43] J. M. Zhi, R. F. Chen, J. Wang, X. Y. Jiao, and R. R. Zhao, "Comparative studies of vasodilating effects of angiotensin(1-7) on the different vessels," Acta physiologica Sinica, vol. 56, no. 6, pp. 730-734, 2004.

[44] W. O. Sampaio, R. A. S. Dos Santos, R. Faria-Silva, L. T. Da Mata Machado, E. L. Schiffrin, and R. M. Touyz, "Angiotensin(1-7) through receptor Mas mediates endothelial nitric oxide synthase activation via Akt-dependent pathways," Hypertension, vol. 49, no. 1, pp. 185-192, 2007.

[45] W. O. Sampaio, C. H. De Castro, R. A. S. Santos, E. L. Schiffrin, and R. M. Touyz, "Angiotensin-(1-7) counterregulates angiotensin II signaling in human endothelial cells," Hypertension, vol. 50, no. 6, pp. 1093-1098, 2007.

[46] R. M. Touyz, "Reactive oxygen species and angiotensin II signaling in vascular cells-implications in cardiovascular disease," Brazilian Journal of Medical and Biological Research, vol. 37, no. 8, pp. 1263-1273, 2004.

[47] C. Szabó, "Role of nitrosative stress in the pathogenesis of diabetic vascular dysfunction," British Journal of Pharmacology, vol. 156, no. 5, pp. 713-727, 2009. 
[48] A. S. De Vriese, T. J. Verbeuren, J. Van De Voorde, N. H. Lameire, and P. M. Vanhoutte, "Endothelial dysfunction in diabetes," British Journal of Pharmacology, vol. 130, no. 5, pp. 963-974, 2000.

[49] E. J. Freeman, G. M. Chisolm, C. M. Ferrario, and E. A. Tallant, "Angiotensin-(1-7) inhibits vascular smooth muscle cell growth," Hypertension, vol. 28, no. 1, pp. 104-108, 1996.

[50] E. A. Tallant and M. A. Clark, "Molecular mechanisms of inhibition of vascular growth by angiotensin-(1-7)," Hypertension, vol. 42, no. 4, pp. 574-579, 2003.

[51] W. B. Strawn, C. M. Ferrario, and E. A. Tallant, "Angiotensin(1-7) reduces smooth muscle growth after vascular injury," Hypertension, vol. 33, no. 1, pp. 207-211, 1999.

[52] B. Langeveld, W. H. Van Gilst, R. A. Tio, F. Zijlstra, and A. J. M. Roks, "Angiotensin-(1-7) attenuates neointimal formation after stent implantation in the rat," Hypertension, vol. 45, no. 1, pp. 138-141, 2005.

[53] D. H. Walter, M. Cejna, L. Diaz-Sandoval et al., "Local gene transfer of phVEGF-2 plasmid by gene-eluting stents: an alternative strategy for inhibition of restenosis," Circulation, vol. 110, no. 1, pp. 36-45, 2004.

[54] B. E. Langeveld, R. H. Henning, B. J. G. L. De Smet et al., "Rescue of arterial function by angiotensin-(1-7): towards improvement of endothelial function by drug-eluting stents," Netherlands Heart Journal, vol. 16, no. 9, pp. 293-298, 2008.

[55] I. Kucharewicz, R. Pawlak, T. Matys, D. Pawlak, and W. Buczko, "Antithrombotic effect of captopril and losartan is mediated by angiotensin-(1-7)," Hypertension, vol. 40, no. 5, pp. 774-779, 2002.

[56] S. Tesanovic, A. Vinh, T. A. Gaspari, D. Casley, and R. E. Widdop, "Vasoprotective and atheroprotective effects of angiotensin (1-7) in apolipoprotein E-deficient mice," Arteriosclerosis, Thrombosis, and Vascular Biology, vol. 30, no. 8, pp. 1606-1613, 2010.

[57] D. Young, G. Waitches, and C. Birchmeier, "Isolation and characterization of a new cellular oncogene encoding a protein with multiple potential transmembrane domains," Cell, vol. 45, no. 5, pp. 711-719, 1986.

[58] R. A. S. Santos, A. C. Simoes e Silva, C. Maric et al., "Angiotensin-(1-7) is an endogenous ligand for the $G$ proteincoupled receptor Mas," Proceedings of the National Academy of Sciences of the United States of America, vol. 100, no. 14, pp. 8258-8263, 2003.

[59] R. A. S. Santos, M. J. Campagnole-Santos, N. C. V. Baracho et al., "Characterization of a new angiotensin antagonist selective for angiotensin-(1-7): evidence that the actions of angiotensin-(1-7) are mediated by specific angiotensin receptors," Brain Research Bulletin, vol. 35, no. 4, pp. 293-298, 1994.

[60] L. M. Cangussu, U. G. M. de Castro, R. D. P. Machado et al., "Angiotensin-(1-7) antagonist, A-779, microinjection into the caudal ventrolateral medulla of renovascular hypertensive rats restores baroreflex bradycardia," Peptides, vol. 30, no. 10, pp. 1921-1927, 2009.

[61] C. R. Tirapelli, S. Y. Fukada, M. A. F. de Godoy, and A. M. de Oliveira, "Analysis of the mechanisms underlying the vasorelaxant action of angiotensin II in the isolated rat carotid," Life Sciences, vol. 78, no. 23, pp. 2676-2682, 2006.

[62] F. Zhang, Y. Hu, Q. Xu, and S. Ye, "Different effects of angiotensin ii and angiotensin-(1-7) on vascular smooth muscle cell proliferation and migration," PLoS ONE, vol. 5, no. 8, Article ID e12323, 2010. 


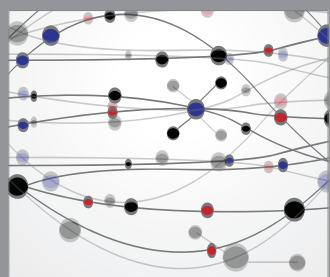

The Scientific World Journal
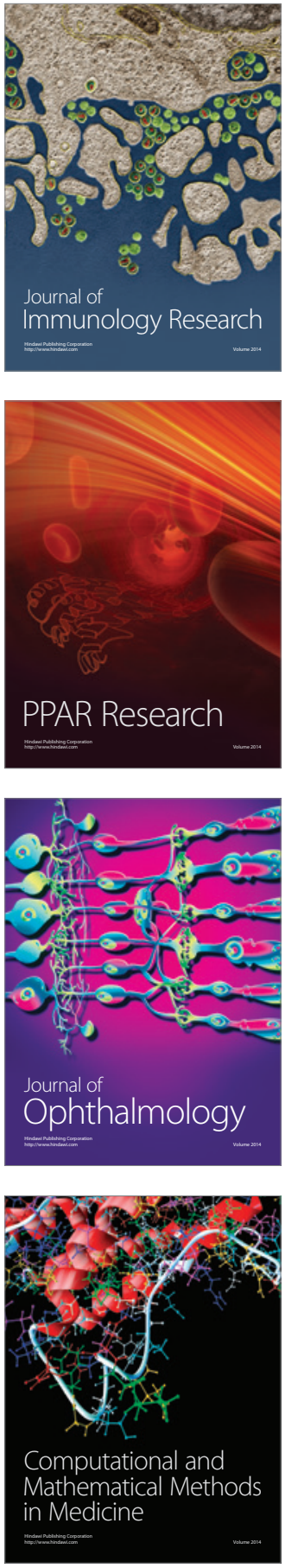

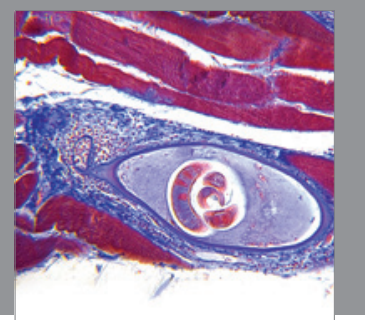

Gastroenterology

Research and Practice
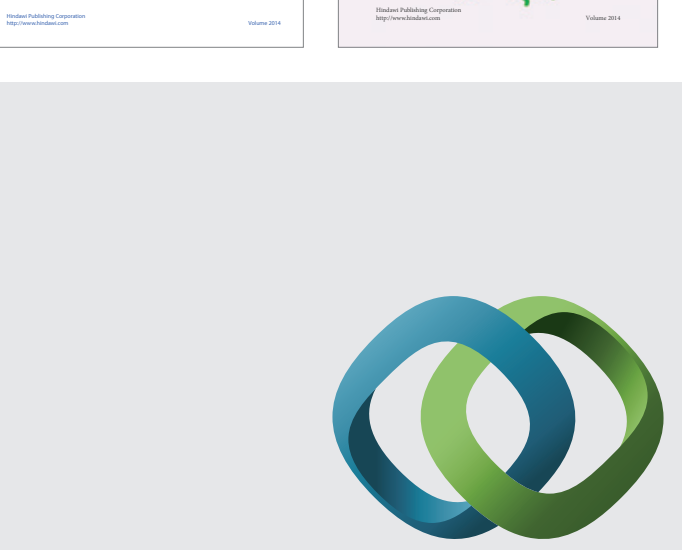

\section{Hindawi}

Submit your manuscripts at

http://www.hindawi.com
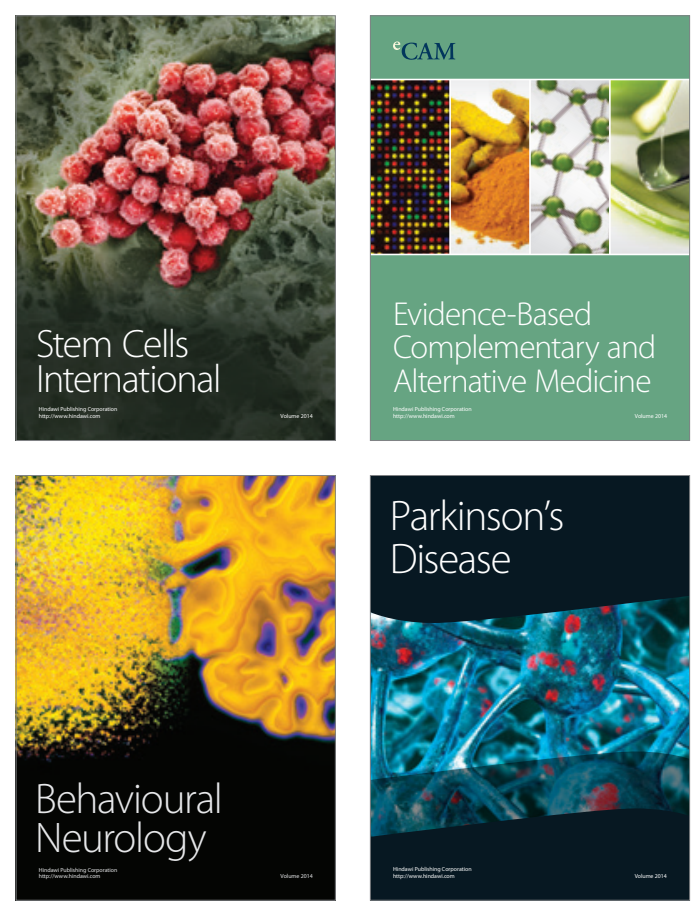

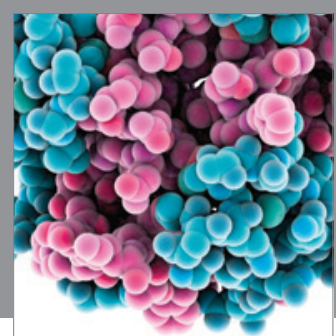

Journal of
Diabetes Research

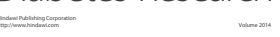

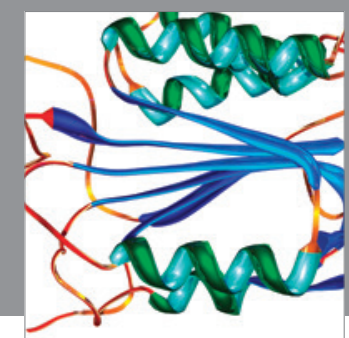

Disease Markers
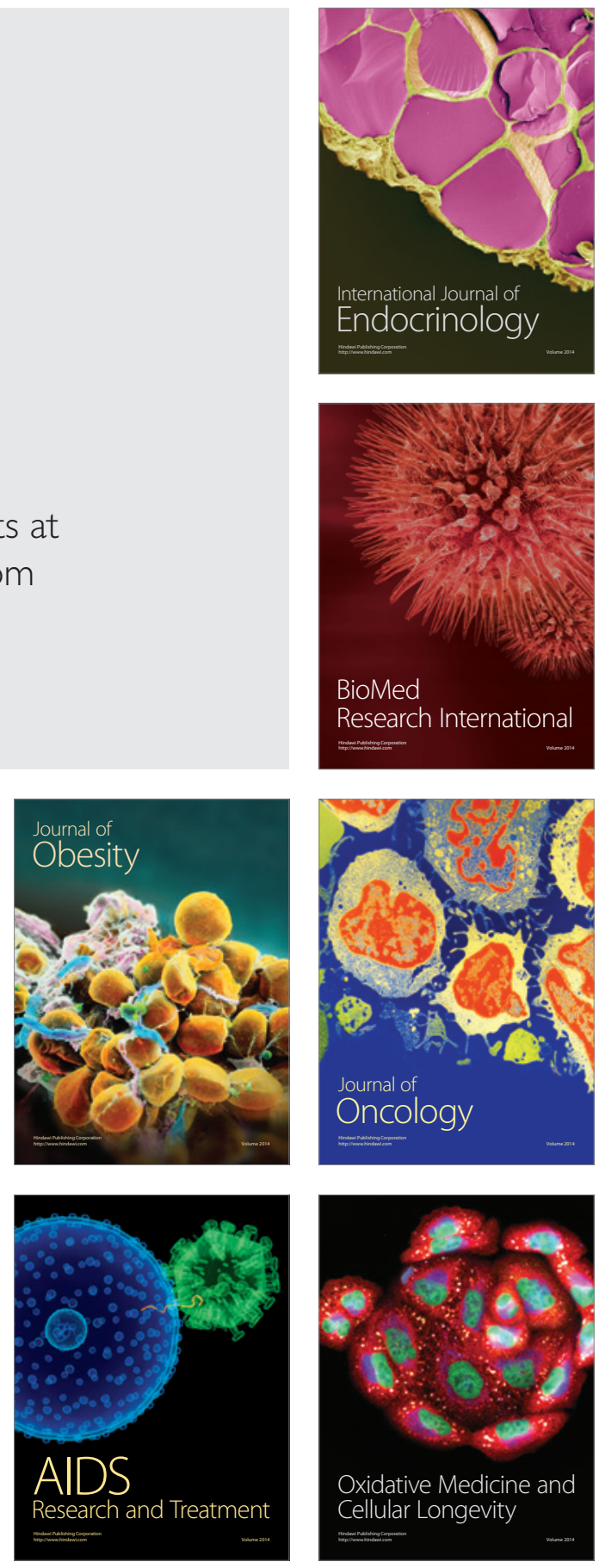